\title{
Characterization of Strombolian events by using independent component analysis
}

\author{
A. Ciaramella ${ }^{1}$, E. De Lauro ${ }^{2}$, S. De Martino ${ }^{2,3,4}$, B. Di Lieto ${ }^{2}$, M. Falanga ${ }^{2,3,4}$, and R. Tagliaferri ${ }^{1}$ \\ ${ }^{1}$ Dipartimento di Matematica ed Informatica, Salerno University, via S. Allende, I-84081 Baronissi (SA), Italy \\ ${ }^{2}$ Dipartimento di Fisica, Salerno University, via S. Allende, I-84081 Baronissi (SA), Italy \\ ${ }^{3}$ INFM, unità di Salerno, via S. Allende, I-84081 Baronissi (SA), Italy \\ ${ }^{4}$ INFN, gruppo collegato di Salerno, via S. Allende, I-84081 Baronissi (SA), Italy
}

Received: 16 June 2004 - Revised: 13 October 2004 - Accepted: 14 October 2004 - Published: 21 October 2004

Part of Special Issue "Nonlinear analysis of multivariate geoscientific data - advanced methods, theory and application"

\begin{abstract}
We apply Independent Component Analysis (ICA) to seismic signals recorded at Stromboli volcano. Firstly, we show how ICA works considering synthetic signals, which are generated by dynamical systems. We prove that Strombolian signals, both tremor and explosions, in the high frequency band $(>0.5 \mathrm{~Hz})$, are similar in time domain. This seems to give some insights to the organ pipe model generation for the source of these events. Moreover, we are able to recognize in the tremor signals a low frequency component $(<0.5 \mathrm{~Hz})$, with a well defined peak corresponding to $30 \mathrm{~s}$.
\end{abstract}

\section{Introduction}

The classical procedure to construct physical models is simple and well established. Firstly, rough but explanatory models are inferred from phenomenology. At this preliminary stage, many possible intuitive pictures live together. They are used to reproduce, on laboratory scale, all the relevant observational aspects. The experiments are, in general, reproducible and some physical laws can be established. These seminal models are improved and discriminated according to their capability to provide new phenomena to test experimentally. There are, however, physical systems for which it is impossible to proceed in this way. In fact, even simple phenomenological aspects cannot be reproduced on laboratory scale, both for practical and conceptual reasons. These systems are characterized by strong nonlinear behaviour that involves many time and spatial length scales.

Geophysical systems belong to this class. In this and similar cases, people speak about observational data rather than experimental data. Sequences, signals, messages, texts, configurations are examples of observational data. Among them,

Correspondence to: M. Falanga

(rosfal@sa.infn.it) an important role is played by the observations (measurements) made in the course of the time, i.e. scalar time series. We can associate, in a natural way, the concepts of complexity, statistics, ergodicity to these series in order to distinguish them, quantitatively. All the sequences can be described, in a formal way, by the unifying concept of Dynamical System (DS). Many theorems and mathematical devices have been elaborated to study DSs. However, they give powerful methods to study asymptotic properties. In the real experimental cases, we start from finite sequences, sometimes very long, and we want to obtain the model. The problem is to extract relevant properties from one or more of the available scalar finite sequences. Then, numerical analysis arises to understand all important parts of information included within series. A review of many numerical methods of nonlinear signal processing developed in the recent years can be found in Abarbanel (1996).

In many cases, sequences can contain information relative to different DSs or sources or signals: therefore a preliminary step is to recognize independent components. The methods based on information theory are natural to use in this research field. The concept of entropy plays a particular role: it is very general and gives a powerful methodology to distinguish the complexities associated to different DSs.

Independent Component Analysis (ICA) is an entropy based technique, useful to separate mixtures of signals (for more details see Hyvärinen et al. (2001) and many papers therein cited). ICA was introduced in the early 1980s in a neurophysiological setting (Hérault, 1984). The introduction of FastICA algorithm (Hyvärinen and Oja, 1997) contributed to the application of ICA to large scale problems due to its computational efficiency.

In the next section, we shall give the mathematical setting of ICA. Here, we consider the problem to understand the behaviour of ICA method with respect to scalar time series generated by geophysical systems. Since they can be 
interpreted as time evolution of a suitable dynamical system, we start clarifying the performance of ICA when it is applied to known DSs. We select different DSs equations representative of large classes, namely linear, nonlinear in the regime of limit cycle, and stochastic DSs, and we use ICA to make separation of the generated synthetic signals. After this section, we show the application of ICA to observational recorded time series of Stromboli volcano.

\section{Independent component analysis}

ICA is a method to find underlying factors or components from multivariate (multidimensional) statistical data.

It is a well defined method in speech context, to solve the classical problem of cocktail party. Imagine to have some people speaking in a room and some microphones recording their voice. The goal of ICA is to extract, from the mixtures of voices, step by step, each independent speaker.

Let us explain in brief the mathematical setting on which ICA is based. We can suppose to have $m$ different recorded time series $\boldsymbol{x}$, that we hypothesize to be the linear superposition of $n$ mutually independent unknown sources $s$, due to different mixing, represented by a constant unknown $m \times n$ matrix A. This mixing is essentially due to path, noise, instrumental transfer-functions, etc. The hypothesis is to have instantaneous linear mixtures of some independent dynamical systems. If the mixing has to be linear, nothing is assumed with respect to the sources, which can be linear or nonlinear.

Formally, the mixing model is written as

$\boldsymbol{x}=\mathbf{A} \boldsymbol{s}+\mu$.

The term $\mu$ takes into account the presence of an additive noise, often omitted in Eq. (1), because it can be incorporated in the sum as one of the source signals. In addition to the source independence request, ICA assumes that the number of available different mixtures $m$ is at least as large as the number of sources $n$. Under these hypothesis, the ICA goal is to obtain a separating matrix $\mathbf{W}=\left(\boldsymbol{w}_{1}, \ldots, \boldsymbol{w}_{m}\right)^{T}$, inverse of $\mathbf{A}$, in such a way that the vector

$y=\mathrm{W} x$

is an estimate $\boldsymbol{y} \simeq \boldsymbol{s}$ of the original independent source signals. We have to remark that the hypothesis of instantaneous mixtures means that we are able to extract "modes" having the same velocity. As a consequence, no delay among the recordings is allowed. In presence of delay, as we will see in the Sect. 4, it is required to align the recorded signals.

We stress that ICA is a statistical model. In other words, from the central limit theorem, we know that the distribution of a sum of independent random variables tends towards a gaussian distribution, under certain conditions. The main idea of the ICA model is to maximize the non-Gaussianity (super-Gaussianity or sub-Gaussianity) to extract the independent components. The separation is achieved on the basis of their statistical independence, evaluated by using 4 th order statistical properties.

In the following, we shall use the fixed-point algorithm, namely FastICA (Hyvärinen and Oja, 1997). The FastICA learning rule finds a direction, i.e. a unit vector $\boldsymbol{w}$ such that the projection $\boldsymbol{w}^{T} \boldsymbol{x}$ maximizes independence of the single estimated source $y$. Independence is here measured by the approximation of the negentropy, that is a measure of nonGaussianity, given by

$J_{G}(\boldsymbol{w})=\left[E\left\{G\left(\boldsymbol{w}^{T} \boldsymbol{x}\right)\right\}-E\{G(v)\}\right]^{2}$,

where $\boldsymbol{w}$ is an $m$-dimensional (weight) vector, $\boldsymbol{x}$ represents our mixture of signals, $E\{\cdot\}$ indicates the expectation value, $E\left\{\left(\boldsymbol{w}^{T} \boldsymbol{x}\right)^{2}\right\}=1$ means variance set to $1, G$ is a suitable approximating contrast function, $v$ is a standardized Gaussian random variable.

Maximizing $J_{G}$ allows to find "one" independent component, or projection pursuit direction. The algorithm requires a preliminary whitening of the data: the observed variable $\boldsymbol{x}$ is linearly transformed to a new variable $\boldsymbol{v}$ with zero-mean and unit variance. Whitening can always be accomplished by e.g. Principal Component Analysis (PCA). This method involves a mathematical procedure that transforms a number of (possibly) correlated variables into a (smaller) number of uncorrelated variables called principal components. The first principal component accounts for as much of the variability in the data as possible, and each succeeding component accounts for as much of the remaining variability as possible. Traditionally, principal component analysis is performed on covariance matrix (Bishop, 1995).

The one-unit "fixed-point" algorithm for finding a row vector $\boldsymbol{w}$ is

$$
\begin{aligned}
\boldsymbol{w}_{i}^{*} & =E\left[\boldsymbol{v} g\left(\boldsymbol{w}_{i}^{T} \boldsymbol{v}\right)\right]-E\left[g^{\prime}\left(\boldsymbol{w}_{i}^{T} \boldsymbol{v}\right)\right] \boldsymbol{w}_{i} \\
\boldsymbol{w}_{i} & =\boldsymbol{w}_{i}^{*} /\left\|\boldsymbol{w}_{i}^{*}\right\|,
\end{aligned}
$$

where $g(\cdot)$ is a suitable nonlinear function, in our case $g(y)=\tanh (y)$, and $g^{\prime}(y)$ is its derivative with respect to $y$. This nonlinear function is introduced to analyse nonlinear time series by means of high-order statistics. The cited algorithm can be generalized to estimate several independent components, which have to be orthonormal one to each other.

ICA is considered a generative model. This means that it describes how the observed data are generated by a process of mixing the components. The basic ICA model, summarizing, requires the following assumptions:

- all the independent components, with the possible exception of one component, must be non-Gaussian (Bell and Sejnowski, 1995; Karhunen, 1996);

- the number of the observed linear mixtures must be at least as large as the number of independent components;

- the matrix A must be of full column rank. 
a)
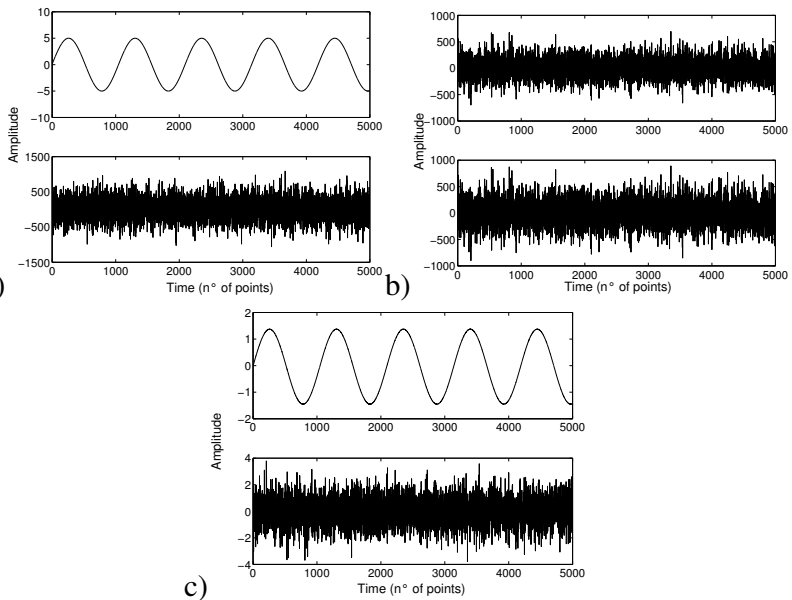

Fig. 1. Separation of a mixture of an harmonic oscillator $(0.01 \mathrm{~Hz}$ frequency) and Gaussian noise (SNR is $-20 \mathrm{db}$ ); time (in number of points) is on $\mathrm{x}$-axis, dimensionless amplitude is on y-axis: (a) original sources; (b) mixed signals; (c) separated signals.

Moreover, ICA contains the following ambiguities or indeterminacies:

- we cannot determine the variances (proportional to energies) of the independent components;

- we cannot determine the order of the independent components.

We have used the basic model of ICA. There are, however, many generalizations born along the time. Several approaches have been proposed to apply ICA when the number of mixtures is less than the number of sources (Roweis, 2000; Jang and Leen, 2003). Other approaches are introduced to accomplish separation of convolved signals (signals with delays and echoes). Moreover, another interesting extension of the ICA could be used in the case of nonlinear mixing (Pajunen and Karhunen, 1997; Hyvärinen and Pajunen, 1999). In the next section, to show the effectiveness of basic ICA for linear mixing, we consider the ICA application to some classes of DSs.

\section{Dynamical systems}

DSs can be considered a general representation of large class of sequences. Then, it is very interesting and explicative as a first step to apply ICA to DSs. We consider, in particular, three types of DSs: linear, nonlinear in the regime of limit cycle, and stochastic systems, taking into account both DSs with few and infinite degrees of freedom. The linear case makes clear how ICA works. Limit cycle and stochastic systems have characteristic behaviour that can be found in many geophysical systems. We have restricted our analysis to linear/nonlinear systems which are linearly superposed. We exclude nonlinear superpositions from our analysis. They have to be treated using a different methodology (Hyvärinen

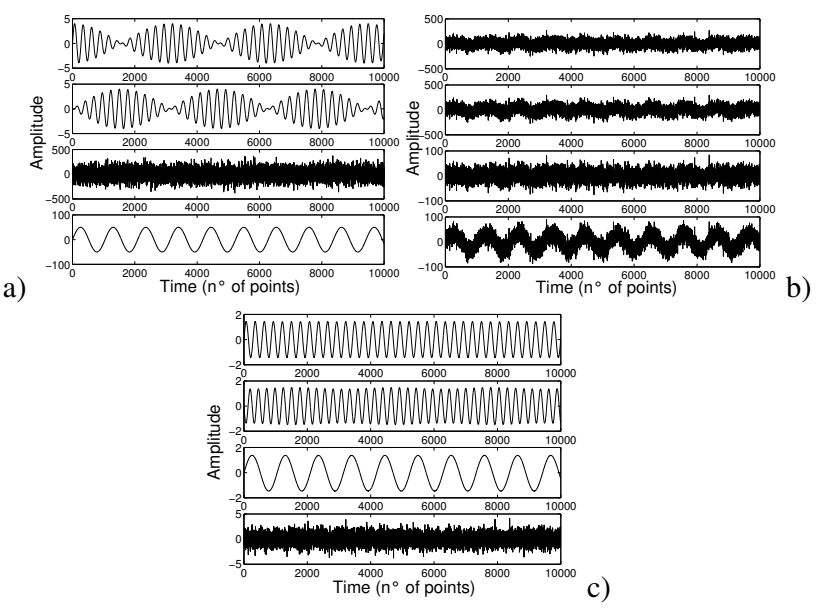

Fig. 2. Separation of the mixture of two coupled harmonic oscillators, one harmonic oscillator and Gaussian noise: (a) source signals; (b) mixed signals; (c) separated signals.

and Pajunen, 1999). In fact, we have not considered nonlinear systems developing chaotic behaviour as for example Rössler attractor, in which the z-component is not well separated because of the nonlinear contribution of the $\mathrm{xz}$ term (Ciaramella et al., 2002 $2^{1}$ ).

Generally, DSs are described by a first order equation system:

$\frac{d \boldsymbol{x}(t)}{d t}=\boldsymbol{F}(\boldsymbol{x}(t))$,

where $\boldsymbol{F}(\boldsymbol{x}(t))$ is a linear, nonlinear or stochastic field.

In particular for stochastic systems, we consider diffusive stochastic processes described by the following equation:

$d x=[v(x, t)] d t+\varepsilon d W$,

where $v(x, t)$ is a field (drift), $d W$ is a Wiener process and $\varepsilon$ is the diffusion coefficient.

\subsection{Linear dynamical systems}

The linear systems that we analyse are single and coupled harmonic oscillators. It is enough to consider two coupled oscillators, because the behaviour of a system with many oscillators is completely equivalent to the previous one. We have made many different experiments on mixtures generated by these systems. We illustrate:

- the separation of an harmonic oscillator and an additive Gaussian noise;

- the separation of coupled oscillators, in beating regime, one harmonic oscillator and a Gaussian noise.

\footnotetext{
${ }^{1}$ Ciaramella, A., De Lauro, E., De Martino, S., Falanga, M., and Tagliaferri, R.: Independent Component Analysis and Dynamical Systems, Salerno University, J. Mach. Learn. Res., preprint submitted, 2002.
} 
a)

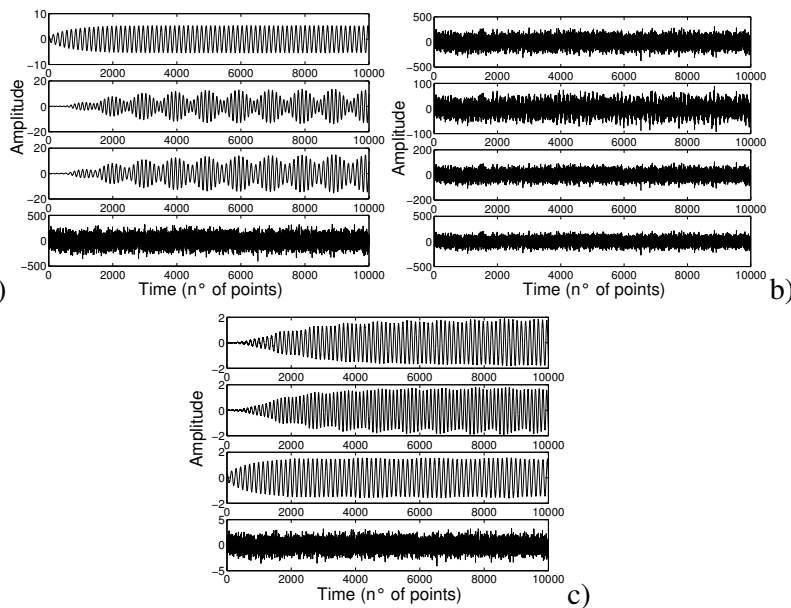

Fig. 3. Separation of the mixtures of two linearly coupled Andronov oscillators, one Andronov oscillator and Gaussian noise: (a) source signals; (b) mixed signals; (c) separated signals.

We can observe in (Fig. 1) how ICA is able to extract the harmonic oscillator from Gaussian noise with a very low signal to noise ratio $(\mathrm{SNR}=-20 \mathrm{db})$. Similar results are achieved even considering different noise.

In Fig. 2, we report the results related to the second experiment. We easily recognize as extracted signals the standard normal modes, a single harmonic oscillator and the additive noise, though the low SNR.

\subsection{Nonlinear dynamical systems}

We consider a particular nonlinear oscillator, i.e. the Andronov oscillator (Andronov et al., 1966). This oscillator is a nonlinear system that generates, with suitable parameters, a limit cycle which is approached asymptotically by all other phase paths. The limit cycle is dynamically stable. It is representative of many nonlinear systems with feedback. As we see in the following, this behaviour can be associated to some interesting natural systems. The equations of the Andronov oscillator with natural frequency $\omega_{0}$, in the suitable form, are:

$\ddot{x}+2 h_{1} \dot{x}+\omega_{0}^{2} x=0$ if $x<b$

$\ddot{x}-2 h_{2} \dot{x}+\omega_{0}^{2} x=0$ if $x>b$.

The nonlinearity is contained in a suitable threshold $b$ ruling the self-coupling. We fix $b=-1 ; h_{1}$ and $h_{2}$ are respectively dissipative and constructive parameters.

By using different parameters and threshold, the Andronov oscillator has different behaviours. In fact, if the threshold is negative, then we can have a limit cycle or forcing oscillations, while, if the threshold is positive, no limit cycle is obtained. In the other cases, for the other parameters, we have:

$-0<h_{1}<h_{2}<1$ : it does not have a limit cycle for any threshold;
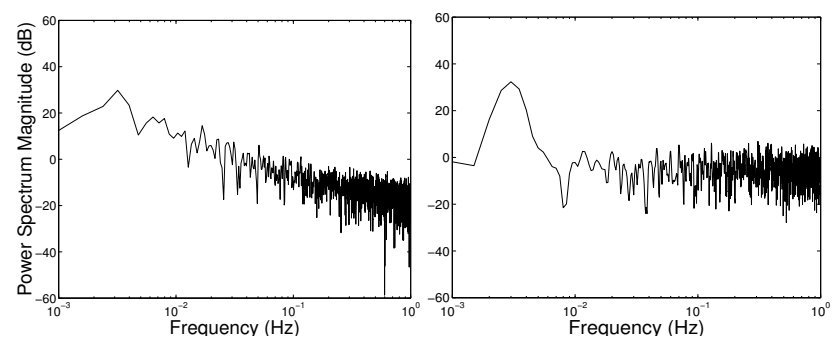

Fig. 4. Power Spectrum Density: (a) a generic story of stochastic process described by Eq. (8); (b) $z(t)$ described by Eq. (9).

$-b=-1$ and $0<h_{2}<h_{1}<1$ : it does have a limit cycle for any initial conditions;

$-b=-1$ e $0<h_{2} h_{1}>1$ : it does have a limit cycle for any initial conditions.

We show a representative example to illustrate the interesting properties of ICA when it is applied to self-oscillating nonlinear systems. In this experiment, we consider two linearly coupled Andronov oscillators with frequency respectively of $0.93 \mathrm{~Hz}$ and $1.1 \mathrm{~Hz}$, one Andronov oscillator with frequency of $0.9 \mathrm{~Hz}$ and Gaussian noise. The SNR in this case is $-20 \mathrm{db}$. Applying the ICA we obtain four separated signals (Fig. 3).

In conclusion linearly coupled Andronov oscillators are well separated by ICA both among them and from superimposed noise. This experiment shows the power of ICA that is able, as in the linear case regarding the normal modes, to extract the independent limit cycles in time domain. We stress that it is not trivial because the nonlinear differential equations cannot be solved and FFT, due to the nonlinearity of the problem, looses its efficacy. If we lower SNR under -20 $\mathrm{db}$ the separation of noise is not so optimal as in linear case.

\subsection{Stochastic systems}

The third class of systems are stochastic diffusive processes. The archetype of models is represented by a simple symmetric bistable potential (double-well) driven by both an additive random noise, i.e. white and Gaussian, and an external periodic bias. Given these features, the response of the system undergoes resonance-like behaviour as a function of the noise level; hence the name stochastic resonance (Gammaitoni et al., 1998). Formally, if we consider the Langevin equation with a small periodic forcing, we obtain:

$d x=\left[x\left(a-x^{2}\right)+A \cos (\Omega t)\right] d t+\varepsilon d W$,

where $d W$ is a Wiener process, i.e. a Gaussian process with zero mean and unitary variance; $x$ is a dimensionless physical variable; $a$ is a parameter related to the double-well potential, $A$ is the amplitude, $\Omega$ the angular frequency of the external periodical forcing, and $\varepsilon$ is the diffusion coefficient.

This system is compared with another that displays a similar frequency content. The latter, denoted as $z(t)$, is described by the following equations: 


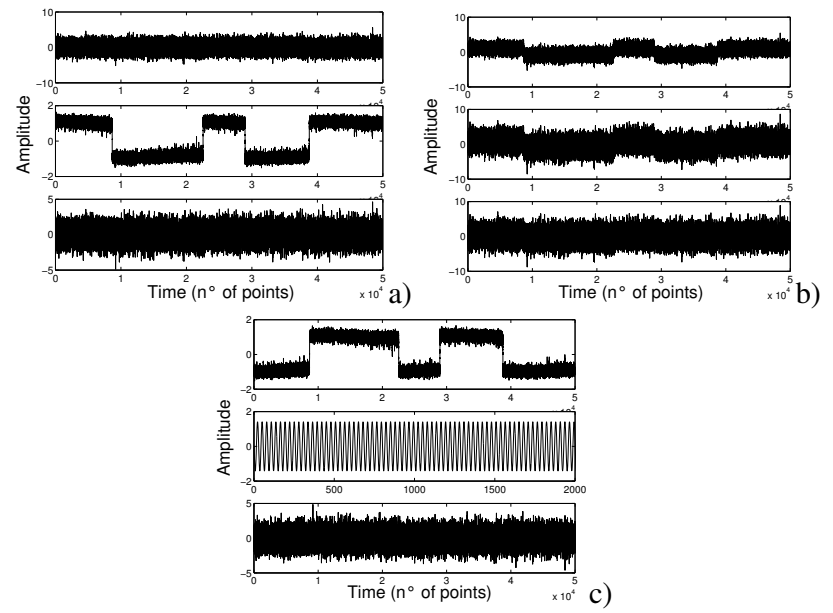

Fig. 5. Separation of the mixture of linear oscillator with frequency of $0.003 \mathrm{~Hz}$ with an additive Gaussian noise and stochastic resonance with the same resonance frequency: (a) source signals; (b) mixed signals; (c) extracted components.

$$
\begin{aligned}
\ddot{x} & =-\Omega^{2} x \\
d y & =\varepsilon d W \\
z(t) & =A x(t)+B y(t),
\end{aligned}
$$

where the first equation is a simple harmonic oscillator with angular frequency $\Omega$; the second is a Wiener process; the third is the superposition of the two, according to the coefficient $A, B$. If we choose $\Omega$ equal to the angular frequency of the stochastic process, in the regime of resonance, we obtain that $z(t)$ has a similar frequency content as $x(t)$ (see Fig. 4).

As one can see in Fig. 5, this case is very impressive, namely the separation is optimal. ICA separates lowdimensional and high-dimensional systems, i.e. harmonic oscillator and both stochastic resonance and Gaussian noise, also in presence of a very similar frequency content. Same results are achieved using different kinds of noise (e.g. uniform noise). Obviously, ICA is not able to recognize the number of degrees of freedom and if we consider real systems for which we have not "a priori" knowledge, we must add to ICA other independent methods (De Martino et al., 2002c)

We should note that linear methods based on FFT fail, because they do not distinguish the systems underlying our mixtures, describing the observed spectra as due to the same DS. In that framework, a stochastic resonance is not at all different from a simple oscillator with noise. The ICA well distinguishes the case in which the harmonic oscillator is linearly superposed to noise from the case in which the FFT peak is generated by a stochastic behaviour, i.e. stochastic resonance.

Now we can draw our conclusions about the explicative synthetic examples. We have applied ICA to some dynamical systems; firstly to linear and nonlinear systems with few degrees of freedom, and then to infinite degrees of freedom

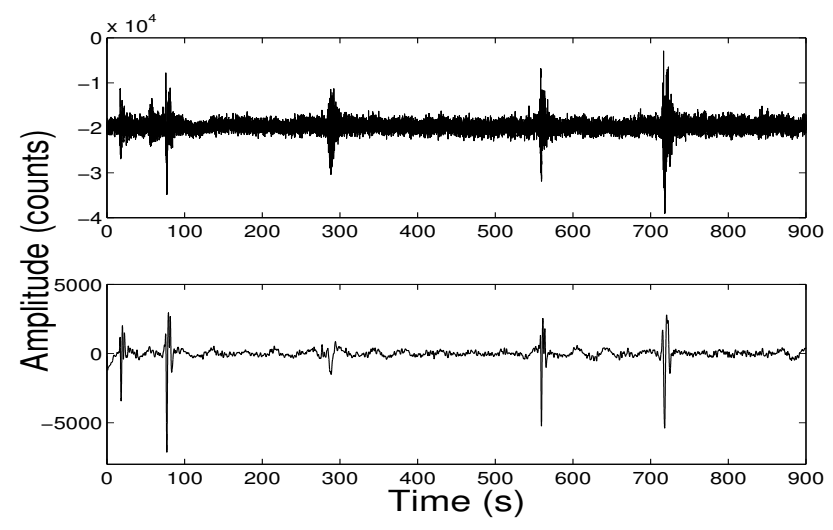

Fig. 6. In the upper figure, 15 minutes of the vertical component of broadband event is plotted (amplitudes in adimensional unit). It was recorded at $\mathrm{T} 1$ station on 19 September at 22:00, during the experiment performed in 1997, where 21 three-component broadband seismometers were deployed on the flanks of the volcano, grouped into three rings at different levels: Top, Middle, Bottom stations. In the lower figure, the same event is filtered in the band $0.02-0.5 \mathrm{~Hz}$, in order to make evident the waveform of the explosions superposed to background tremor.

systems, namely diffusion processes in the regime of stochastic resonance.

Regarding the linear systems, we have obtained optimal separation from very high superposed noise. Furthermore, the ICA acts as Fast Fourier Transform but in time domain since it gives us the normal modes of the system.

The performance of ICA is valuable also in the case of nonlinear systems where we separate coupled Andronov oscillators, not trivial from the point of view of dynamical systems. Also in these experiments, the separation from noise is well made. The experiments with stochastic resonance are very impressive: the superposed periodic and stochastic signals are completely separated, i.e. ICA perfectly recognizes the different superposed dynamical systems also when the Fourier Transform is irresolute (insensitive). As a conclusive remark, we can say that ICA is a method to apply "a priori" as a pre-analysis to scalar experimental series, namely it allows to recognize if the scalar series contain one or more independent signals.

\section{Application of ICA to Strombolian events}

We study the seismic signals recorded at Stromboli volcano. This volcano is characterized by basaltic eruptions. In these eruptions, the relative motion of gas with respect to the fluid produces either an annular flow (Hawaian Fire Fountains) or a Slug flow (Strombolian explosions). In fact, the typical seismic signature of Stromboli is the continuous volcanic tremor (continuous vibration of the ground around the volcano) due to degassing to which repeating explosion quakes are superposed (see Fig. 6). 

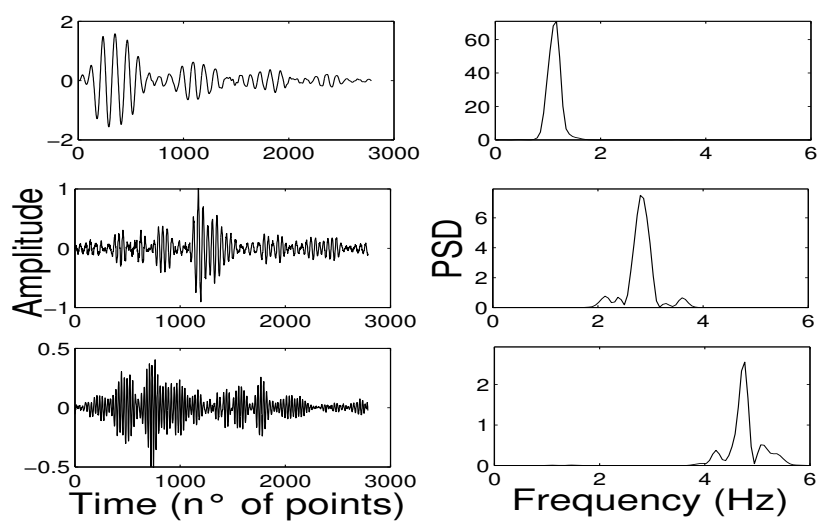

Fig. 7. Explosions in the band greater than $0.5 \mathrm{~Hz}$ : Denoised independent components of radial direction of motion and their spectra (sampling frequency equal to $125 \mathrm{~Hz}$; amplitude in adimensional unit).

Tremor and explosions have a very similar frequency content. In fact, both the behaviours are generated by complex processes of magma flow and turbulent degassing.

Despite many studies (e.g. Chouet et al., 1997, 1999, 2003), the dynamics underlying the generation of these behaviours is not yet well understood.

In our studies, we have applied ICA to Strombolian events considering both short-period $(0.5-50 \mathrm{~Hz})$ and broadband $(0.02-50 \mathrm{~Hz})$ recorded seismograms. Strombolian signals, due to their stationarity, are suitable to apply ICA. The aim is to decompose, if it is possible, recorded series into statistically independent components. In this way, we get information about the "modes" involved in the full dynamics and constrain source geometry and mechanism. The analysis will be carried on explosions and tremor, separately.

In order to avoid any delay among recording stations located in different places, the seismic traces are aligned using the cross-correlation function. This satisfies the ICA request of instantaneous mixing.

To get other information, we have also adopted different techniques. They consist in techniques generally used in nonlinear signal processing, and well-known or innovative methods to investigate seismological signals. In particular, we have applied parametric and non parametric spectral analysis; nonlinear denoising techniques (Kostelich and Schreiber, 1993); particle motion and polarization filtering (Kanasewich, 1981); methods to reconstruct phase space starting from scalar time series (estimate of the dimension (Grassberger and Procaccia, 1983), Average Mutual Information (Fraser and Swinney, 1986), False Nearest Neighbors (Kennel et al., 1992)); trajectory space analysis to estimate the variety of dynamical systems presents in the data (Paladin and Vulpiani, 1987).

As regards explosions at high frequency, we report the results obtained decomposing signals recorded by using shortperiod seismometers (Chouet et al., 1998). ICA has been applied to explosions recorded by seismometers along the three
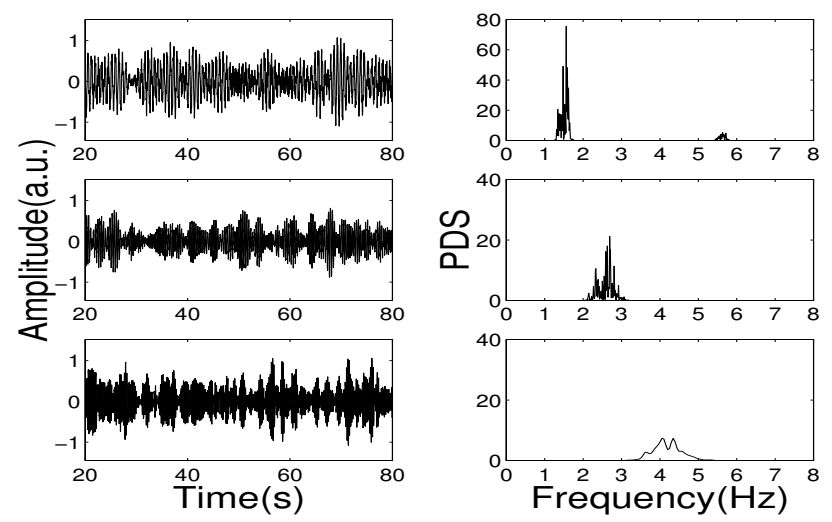

Fig. 8. Denoised independent components of tremor, by ICA, in the range $>0.5 \mathrm{~Hz}$ related to the vertical direction and their spectra (amplitude in adimensional unit).

orthonormal directions of motion, i.e. radial, transverse, vertical with respect to the crater area.

We display in Fig. 7 the results of the radial direction; the other directions show a similar behaviour (Acernese et al., 2003). As one can see (Fig. 7), the wavefield is the linear superposition in time domain of three independent components, characterized by well defined and separate frequency bands (respectively $0.8-1.2,2.4-3.0,3.2-4.5 \mathrm{~Hz}$ ).

The first two bands present wavefield mainly composed of body waves with radial polarization, pointing towards the crater area. In the last band, the very low SNR, together with the corresponding short wavelengths, does not allow to individuate a defined direction (Acernese et al., 2004).

Similar results are achieved analysing broadband explosions. In addition, in this case, we extract also a component corresponding to the VLP signal (Falanga, 2003) as already observed by Chouet et al. (2003).

The reconstruction of the phase space establishes that explosions are associated to a low-dimensional dynamical system characterized by dimensions in the range [2-3] (De Martino et al., 2002a).

Then, trajectory space analysis, performed on broadband signals, states that explosions are generated by an unique dynamical system (De Martino et al., 2004), though Chouet et al. (2003) have found two distinct kinds of explosions, which have been associated to the two distinct vents at Stromboli in 1997. The differences between the two types of events are related more to slight variations in conduit geometries rather than differences in the dynamics generation of the phenomena.

We have also analysed, as already said, the tremor. In this case, it is convenient to consider separately two frequency bands ( $>0.5 \mathrm{~Hz}$ and $<0.5 \mathrm{~Hz}$ ). Namely, the low frequency band can contain waves travelling with different velocity with respect to the high frequency wavefield. In Fig. 8, as you can see, tremor shows ICA extracted components similar to explosion quakes, in waveform and frequency content. Of course, regarding tremor, the individuation of clear bands is 

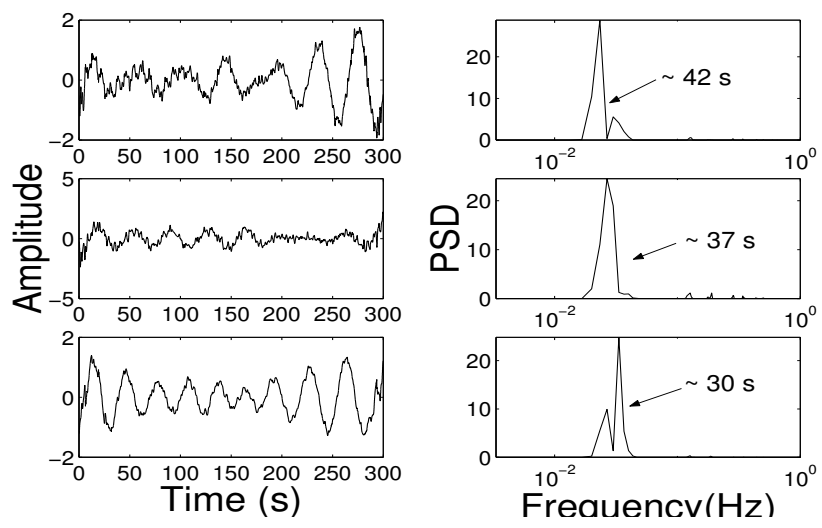

Fig. 9. Denoised independent components by ICA in the range $(0.02-0.5 \mathrm{~Hz})$ of vertical direction of motion and their spectra (amplitude in adimensional unit).

more difficult due to very low SNR. Polarization analysis on tremor has already been performed on short-period recorded signals (Chouet et al., 1997). We are extending the analysis to broadband signals as extracted by ICA. This will be matter of a forthcoming paper.

All the results persuade us to think that, regarding high frequency content, the superficial source is stationary and not destructive. The wavefield is generated by the excitation of only few degrees of freedom of the complex fluid-dynamical source system.

Possible models of the production of these oscillations have been postulated by Julian (1994), Ida (1996) and James et al. (2004). In particular, Julian (1994) suggested an organ pipe model, with a constant rate supply of fluids inside a cylinder conduit for a variety of almost periodic signals observed on volcanoes.

The independent component analysis of organ pipe acoustic emission by Bottiglieri et al. (2004) seems to support this model. Namely, in an organ pipe, a constant rate supply of pressure produces self-sustained sounds and ICA is able to recognize exactly three independent components in limit cycle regime, corresponding to a fundamental Landau mode and two excited ones. In this case, due to the cylindrical symmetry of the pipe the waveforms are simpler than in Stromboli case.

In the scheme of organ pipe model for Stromboli volcano, the tremor is the basic signal; explosions are generated by an enhancing in amplitude of tremor. The enhancing may be due to the formation of slug within the shallow pumping system. In order to get information about the generative process of slugs, i.e. coalescence phenomenon, we focalise our attention on tremor at very low frequency band $(0.02-0.5 \mathrm{~Hz})$. We report in Fig. 9 the extracted components by means of ICA related to windows containing obviously only tremor. As we can see, three components in the band 30-42 s appear, with a fundamental peak corresponding to a $30 \mathrm{~s}$ periodicity (De Martino et al., 2002b, 2003; Falanga, 2003).

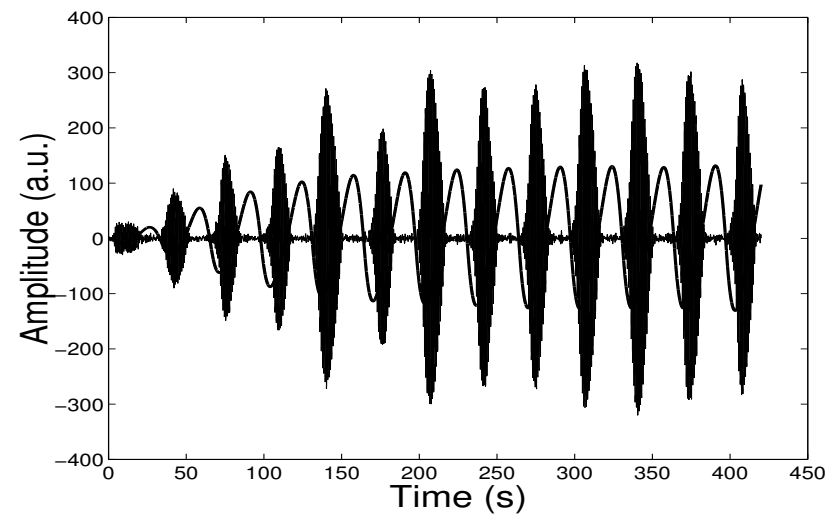

Fig. 10. Toy-model: time evolution of a limit cycle with time variable threshold; in the same figure, time evolution of threshold is also plotted (bold line describing a limit cycle).

Polarization analysis, performed on few tremor signals, filtered in a very low frequency band $(<0.1 \mathrm{~Hz})$ in order to investigate the nature of the 30-40 s extracted components, shows, as a preliminary result, a wavefield polarized in transverse direction with respect to crater area. Further investigation, that takes into account all the statistics, is under study.

Finally, we have estimated the apparent velocity of this component. We obtain values of velocity very low of the order of $100 \mathrm{~m} / \mathrm{s}$. The latter suggests that we are dealing with a subsonic slow wave originating in crater area.

Oscillations, in this low frequencies regime, have been observed already on other volcanoes (e.g. Aster et al., 2003). They have been associated to the formation of slug within the magmatic system. So, they have been recognized as gravity waves although the sizes of slug are not well estimated (e.g. Aster et al., 2003).

\section{Conclusions}

We have applied ICA to Strombolian seismological signals as an "a priori" tool. Combining ICA with other methods, we have obtained many constrains to model Strombolian activity source. ICA gives five "modes" directly in the time domain. This means that, though in a suitable coupling limit, Stromboli recorded signals are the linear superposition of these five modes (three at high frequency, VLP and 30-40 s mode). Then, the physical model must reproduce these "modes".

Our approach clarifies the situation of high frequencies $(>0.5 \mathrm{~Hz})$ of Stromboli: in this range, we are in presence of vibrations of volcanic conduit induced by permanent degassing. We prove that tremor and explosions display not only a similar frequency content, but also similar waveforms in time domain. A pregnant difference is the amplitude enhancing.

Analogies with organ pipe model are possible once having shown that strombolian high frequency wavefield can be decomposed into three separated frequency bands. In fact the degassing is almost stationary as the pumping in the organ 
pipe, although the wavefield produced by the vibration of conduit propagates in a more complex medium with respect to the air (as in the case of acoustic field produced by organ pipe). The waveforms are more complex, namely we are dealing with a pipe (conduit) having a more complex geometry than a cylindrical pipe. But, in both cases, we find three self-sustained "modes" produced by a dynamical system in limit cycle regime.

The heart of sound production in organ pipe is the edge. The same function for the generation of ground vibration (the coupling between degassing and the conduit) can be ruled by the variable geometry of the conduit (James et al., 2004).

The ICA has evidenced, also, component at $30 \mathrm{~s}$, that can furnish the means by which the permanent tremor becomes enhanced in the explosion.

We can consider a very simple model to clarify this point. Take one dimensional limit cycle (Eq. 7) with a threshold $b$ depending on time (see Fig. 10). The threshold in the limit cycle acts as a sort of potential ruling the contributes coming from pumping and dissipation, such a way it represents the stationary degassing and all the dissipative effects surely present in a volcanic structure. When the formation of slug occurs, there is a time variation of pumping so that the system goes in a excited level. Due to the fact that time duration of slug formation is very short, the system immediately decays towards the basic energetic state.

This very rough model is constructed to show a possible mechanism of self-interaction to obtain the seismic record associated to explosion, as enhancing of tremor. Namely, the high frequency amplitude enhancing is the effect of the density time variation due to coalescence, i.e. to the formation of slug.

Obviously, the simulation of tremor as a simple limit cycle at the frequency of $1 \mathrm{~Hz}$ and the simulation of low frequency mode as harmonic oscillator with a period of $30 \mathrm{~s}$ are very far from the reality.

For example, we have not considered that many "modes" come within the real system, that there is a neglected intrinsic stochasticity. Moreover the threshold signal, which represents in a rough way the low frequency mode, is by itself nonlinear and stochastic. Finally, the recorded waveforms are due to a complex geometry of conduit and the real system is not at all one-dimensional. Nevertheless, we think that this toy model could be a starting point that deserves further studies to be implemented to become the physical model of Stromboli in the regime of its standard activity.

Edited by: M. Thiel

Reviewed by: two referees

\section{References}

Abarbanel, H. D. I.: Analysis of observed chaotic data, SpringerVerlag, New York Inc., 1996.

Acernese, F., Ciaramella, A., De Martino, S., Falanga, M., and Tagliaferri, R.: Neural Networks for blind sources separation of Stomboli explosion quakes, IEEE Neural., 14, 1, 2003.

Acernese, F., Ciaramella, A., De Martino, S., Falanga, M., Godano, C., and Tagliaferri, R.: Polarisation analysis of the independent components of low frequency events at Stromboli volcano (Eolian Island, Italy), J. Volcanol., Special Issue, edited by: Harris, A., Hort, M., and Ripepe, M., 137, 1-3, 153-168, doi:10.1016/j.jvolgeores.2004.05.005, 2004.

Andronov, A. A., Vitt, A. A., and Khaikin, S. E.: Theory of oscillators, Dover Publication Inc., New York, 1966.

Aster, R., Mah, S., Kyle, P., McIntosh, W., Dunbar, N., Jonson, J., Ruiz, M., and McNamara, S.: Very long period oscillations of Mount Erebus Volcano, J. Geophys. Res.-Sol., 108, B11, 2522, doi:10.1029/2002JB002101, 2003.

Bell, A. J. and Sejnowski, T. J.: An information-maximisation approach to blind separation and blind deconvolution, Neural Comp., 7, 1129-1159, 1995.

Bishop, M. C.: Neural Networks for Pattern Recognition, Clarendon Press-Oxford, 1995.

Bottiglieri, M., De Lauro, E., De Martino, S., and Falanga, M.: Analogical model inferred from time domain analysis and generating organ pipes self sustained-tone, Int. J. Mod. B, 18, 4-5 509-518, doi:10.1142/S0217979204024124, 2004.

Chouet, B. A., Saccorotti, G., Martini, M., Dawson, P., De Luca, G., Milana, G., and Scarpa, R.: Source and path effect in wavefield of tremor and explosions at Stromboli Volcano, Italy, J. Geophys. Res.-Sol., 102, 15129-15150, 1997.

Chouet, B. A., Dawson, P., De Luca, G., Martini, M., Milana, G., Saccorotti, G., and Scarpa, R.: Array analyses of seismic wavefields radiated by eruptive activity at Stromboli volcano, Italy, CNR-Gruppo Nazionale per la Vulcanologia, Felici editore, Ospedaletto, Pisa, 1998.

Chouet, B. A., Saccorotti, G., Dawson, M., Martini, M., Scarpa, R., De Luca, G., Milana, G., and Cattaneo, M.: Broadband measurements of sources of explosions at Stromboli Volcano, Italy, Geophys. Res. Lett., 26, 13, 1937-1940, 1999.

Chouet, B. A., Dawson, P., Ohminato, T., Martini, M., Saccorotti, G., Giudicepietro, F., De Luca, G., Milana G., and Scarpa, R.: Source mechanism of explosions at Stromboli determined from moment tensor inversions of very-long-period data, J. Geophys. Res.-Sol., 108, B1, 2019, doi:10.1029/2002JB001919, 2003.

De Martino, S., Godano, C., and Falanga, M.: Self-sustained oscillator as a model for explosion quakes at Stromboli Volcano, Nonl. Proc. Geophys., 9, 31-35, 2002a,

SRef-ID: 1607-7946/npg/2002-9-31.

De Martino, S., Falanga, M., Godano, C., Scarpa, R., and Tronca, F.: Caratteristiche dei segnali sismici a larga banda di Stromboli, $21^{\circ}$ Convegno Nazionale del Gruppo Nazionale di Geofisica della Terra Solida (GNGTS), Roma, 19-21 Novembre, 2002b.

De Martino, S., Falanga, M., and Mona, L.: Stochastic Resonance Mechanism in Aerosol Index Dynamics, Phys. Rev. L., 89, 12, 2002c. 
De Martino, S., Falanga, M., Godano, C., Scarpa, R., and Tronca, F.: Characteristics of broadband events at Stromboli volcano, EGS-AGU-EGU 2003 Joint Assembly, Nice, France, 6-11 April, 2003.

De Martino, S., Falanga, M., and Godano, C.: Dynamical similarity of explosions at Stromboli volcano, Geophys. J. I., 157, 3, 12471254, doi:10.1111/j.1365-246X.2004.02263.x, 2004.

Falanga, M.: Costruzione di modelli fisici a partire da segnali osservazionali, $\mathrm{PhD}$ thesis, Salerno University, Febbraio 2003.

Fraser, A. M. and Swinney, H. L.: Independent coordinates for strange attractors from mutual information, Phys. Rev. A, 33, 1134-1139, 1986.

Gammaitoni, L., Hänggi, P., Jung, P., and Marchesoni, F.: Stochastic resonance, Rev. Mod. Phys., 70, 1, 223, 1998.

Grassberger, P., and Procaccia, I.: Measuring the strangeness of strange attractors, Physica D, 9, 189-208, 1983.

Hérault, J., and Ans, B.: Circuits neuronaux à synapses modifiables: décodage de messages composites par apprentissage non supervisé, C.-R. de l'Accadémie des Sciences, 299(III-13), 525-528, 1984.

Hyvärinen, and Oja, E.: A fast fixed-point algorithm for independent component analysis, Neural Comp., 9, 7, 1483-1492, 1997.

Hyvärinen, A., Karhunen, J., and Oja, E.: Independent Component Analysis, John Wiley \& Sons, New York, 2001.

Hyvärinen, A. and Pajunen, P.: Nonlinear independent component analysis: existence and uniqueness results, Neural Netw., 12, 3, 429-439, 1999.

Ida, Y.: Cyclic fluid effusion accompanied by pressure change: implication for volcanic eruptions and tremor, Geophys. Res. Lett., 23, 1457-1460, 1996.
James, M. R., Lane, S. J., Chouet, B., Gilbert, J. S.: Pressure changes associated with the ascent and bursting of gas slugs in liquid-filled vertical and inclined conduits, J. Volcanol., 129, 6182, 2004.

Jang, G. J. and Leen, T. W.: A probabilistic Approach to Single Channel Blind Signal Separation, MIPS 2003, 15, MIT Press, 2003.

Julian, B. R.: Volcanic tremor: nonlinear excitation by fluid flow, J. Geo. R.-Sol., 99, 11 859-11 877, 1994.

Kanasewich, E. R.: Time sequence analysis in geophysics, Univ. of Alberta Press, Edmonton-Canada, 1981.

Karhunen, J.: Neural approach to independent component analysis and sources separation. In Proceedings of Fourth European Symposium on Artificial Neural Networks, 249-266, 1996.

Kennel, M. B., Brown, R., and Abarbanel, H. D. I.: Determining embedding dimension for phase space-reconstruction using a geometrical construction, Phys. Rev. A, 45, 3403-3411, 1992.

Kostelich, E. J. and Schreiber, T.: Noise reduction in chaotic time series data: A survey of common methods, Phys. Rev. E, 48, 1752-1763, 1993.

Pajunen, P., and Karhunen, J.: A Maximum Likelihood Approach to Non-Linear Blind Source Separation, Proceedings of the 1997 Int. Conf. on Artificial Neural Networks (ICANN'97), 541-546, Lausanne, Switzerland, 1997.

Paladin, G. and Vulpiani, A.: Anomalous scaling laws in multifractal objects, Phys. Report, 156, 147, 1987.

Roweis, S. T.: One Microphone Separation, Adv. Neural Inf. Process. Syst., 13:793-799, 2000. 